\title{
Hair Follicle Nevus with Sebaceous Hyperplasia: A Dermoscopic Observation
}

\author{
Kazutoshi Harada Yuichiro Kato Yukihiko Kato Ryoji Tsuboi \\ Department of Dermatology, Tokyo Medical University, Tokyo, Japan
}

\section{Key Words}

Hair follicle nevus · Trichofolliculoma · Juvenile xanthogranuloma - Sebaceous hyperplasia .

Dermoscopy

\begin{abstract}
We herein report a case of hair follicle nevus, a rare hamartoma found on the face and showing follicular differentiation, which was associated with sebaceous hyperplasia. Dermoscopy of the lesion showed yellow globules surrounded by crown vessels/telangiectasias and scattered tiny hairs. Histopathological investigation revealed hyperplasia of the sebaceous glands and proliferation of well-differentiated vellus hair follicles. These pathological findings were thought to correspond to the yellowish globules and tiny hairs observed under dermoscopy. Hair follicle nevus associated with sebaceous hyperplasia is extremely rare; however, dermoscopic examination can suggest an appropriate diagnosis. The present case proved the diagnostic usefulness of dermoscopy for cutaneous tumors with hair follicular and sebaceous glandular differentiation.

(C) 2015 The Author(s) Published by S. Karger AG, Basel
\end{abstract}

\section{Introduction}

Hair follicle nevus (HFN) is a rare hamartoma occurring on the face that shows follicular differentiation [1]. Previously, HFN was confused with trichofolliculoma, a benign hamartoma that should be differentiated from HFN. Trichofolliculoma can be distinguished from HFN based on the pathological findings of a central cyst-like structure surrounded by a proliferation of hair follicles [2]. The dermoscopic finding of neither a central opening nor a cyst was useful for the differential diagnosis of HFN and trichofolliculoma [3]. We herein report a case of HFN on the nose with sebaceous hyperplasia with characteristic dermoscopic findings. 
Harada et al.: Hair Follicle Nevus with Sebaceous Hyperplasia: A Dermoscopic Observation

Dermoscopy of the lesion demonstrated yellow globules surrounded by crown vessels/ telangiectasias and scattered tiny hairs. Although there were no previous case reports describing the same dermoscopic findings as in our case, hyperplasia of the sebaceous glands and proliferation of well-differentiated vellus hair follicles were implied by dermoscopy, indicating the diagnostic usefulness of dermoscopy for the cutaneous papular and nodular lesions. The present case proved the diagnostic usefulness of dermoscopy for folliculosebaceous tumor.

\section{Case Report}

An 18-year-old girl presented with a papule on the nose. The lesion had appeared at 8 years of age and had slightly enlarged before her visit. Physical examination revealed a firm, pinkish, dome-shaped papule $4 \mathrm{~mm}$ in diameter (fig. 1). Dermoscopy showed yellow globules surrounded by crown vessels/telangiectasias (fig. 2). Although no central follicular opening was observed, tiny hairs were scattered on the lesion. There were no previous case reports describing the same dermoscopic findings. Thus the skin lesion was completely resected surgically for diagnosis. Histological examination revealed proliferation of welldifferentiated vellus hair follicles in the upper reticular dermis (fig. 3a). Interestingly, hyperplasia of the sebaceous glands connected to the hair follicles was also observed. These pathological findings were thought to correspond to the yellowish globules and tiny hairs scattered over the lesion revealed by dermoscopy. The connective tissue sheath of the tiny follicles presented marked fibrous thickening, and the sebaceous glands in the lower dermis were constituted of mature sebocytes (fig. $3 \mathrm{~b}$ ). No central cyst structure surrounded by a proliferation of hair follicles or cartilage was found on histopathological examination. On the basis of these findings, we made the diagnosis of HFN associated with sebaceous hyperplasia. No recurrence was observed for the following 6 months.

\section{Discussion}

Pippione et al. [4] described HFN as a rare hamartoma consisting of well-differentiated vellus hair follicles. Until then, HFN had often been misdiagnosed as trichofolliculoma, a benign hamartoma characterized by a central follicular opening with secondary hair follicles [2]. HFN usually presents as a papule, plaque or nodule on the face [1]. The lesion is fleshcolored to pinkish and sometimes, though rarely, brown [3]. An unusual form of HFN showing pedunculated, skin-colored cutaneous nodule has been reported [5]. The pathological features of HFN include numerous mature vellus hair follicles abnormally located in the upper dermis and occasionally accompanied by sebaceous or eccrine glands and pilar muscles [2]. This case presented a pinkish papule on the nose and histologically small hair follicles vertically aligned in the upper dermis in accordance with the diagnostic criteria for HFN (fig. 3a). The characteristic features of the present case pointed to sebaceous hyperplasia. In contrast, the hair follicles in the present lesion were immature and very small in comparison with those observed in previous reports of HFN. The progenitor cells in HFN in this case might have preferentially differentiated into sebaceous glands rather than hair follicles.

Histological as well as dermoscopic examination of the papule revealed no central opening, cyst or hair follicle, thus negating the diagnosis of trichofolliculoma. The hyperplasia of the hair follicles and sebaceous glands suggested the diagnosis of folliculosebaceous cystic hamartoma; however, this diagnosis was also excluded due to the absence of a central cystic 
Harada et al.: Hair Follicle Nevus with Sebaceous Hyperplasia: A Dermoscopic Observation

formation. An accessory auricle sometimes demonstrates similar pathological findings to those of HFN. Histologically, the absence of cartilage and the anatomical localization of the lesion may negate the diagnosis of accessory auricle. Ban et al. [6] described that the accessory auricle can be differentiated from HFN by the presence of abundant subcutaneous fat. In accordance with this report, proliferation of fat cells was not observed in the lesion presented here (fig. 3a).

Dermoscopic examination in this case revealed the interesting finding that the yellow globules were surrounded by crown vessels/telangiectasias and that tiny hairs were scattered throughout the lesion (fig. 2). The dermoscopic findings of juvenile xanthogranuloma include yellow globules against an orange-yellow background. This finding was consistent with the dermoscopic findings in the present case. However, the vasculature was different. The vessels in juvenile xanthogranuloma are arborizing, whereas those in HFN, often referred to as crown vessels, are non-arborizing and surround yellow nodules [7]. Although the yellow globular structures associated with crown vessels were dermoscopically revealed in sebaceous hyperplasia, the color of the globules in the lesion was whitish-yellow. The globular structures seen in sebaceous hyperplasia are additionally accompanied by a small crater [8]. These findings could differentiate the present case from sebaceous hyperplasia. Previously the dermoscopic examination of HFN revealed a large number of follicular openings without yellow globules; however, these findings were not consistent with that of the case described here [3]. Although histological examination was required for an accurate diagnosis in the present case, dermoscopy clearly demonstrated the pathological findings, including hyperplasia of the sebaceous glands and proliferation of well-differentiated vellus hair follicles. Thus the present case demonstrated the diagnostic availability of dermoscopy for the cutaneous tumor with hair follicular and sebaceous glandular differentiation.

\section{Statement of Ethics}

The authors have no ethical conflicts to disclose.

\section{Disclosure Statement}

The authors have no conflict of interest to declare.

\section{References}

1 Choi EH, Ahn SK, Lee SH, Bang D: Hair follicle nevus. Int J Dermatol 1992;31:578-581.

$\checkmark 2$ Labandeira J, Peteiro C, Toribio J: Hair follicle nevus: case report and review. Am J Dermatopathol 1996;18:90-93.

3 Okada J, Moroi Y, Tsujita J, Takahara M, Urabe K, Kiryu H, Furue M: Hair follicle nevus - a dermoscopic approach. Eur J Dermatol 2008;18:185-187.

4 Pippione M, Aloi F, Depaoli MA: Hair-follicle nevus. Am J Dermatopathol 1984;6:245-247.

5 Jedrych J, Gehris R, Ho J: Another infant with an extracephalic hair follicle nevus: an under-diagnosed entity? J Cutan Pathol 2015;42:155-157.

6 Ban M, Kamiya H, Yamada T, Kitajima Y: Hair follicle nevi and accessory tragi: variable quantity of adipose tissue in connective tissue framework. Pediatr Dermatol 1997;14:433-436.

7 Unno T, Minagawa A, Koga H, Uhara H, Okuyama R: Alteration of dermoscopic features in a juvenile xanthogranuloma during follow-up of 43 months. Int J Dermatol 2014;53:e590-e591.

-8 Zaballos P, Ara M, Puig S, Malvehy J: Dermoscopy of sebaceous hyperplasia. Arch Dermatol 2005;141:808. 
Case Reports in
Dermatology

Case Rep Dermatol 2015;7:358-362

\begin{tabular}{l|l}
\hline DOI: $10.1159 / 000442969$ & C 2015 The Author(s). Published by S. Karger AG, Basel \\
\hline
\end{tabular} www.karger.com/cde

Harada et al.: Hair Follicle Nevus with Sebaceous Hyperplasia: A Dermoscopic Observation

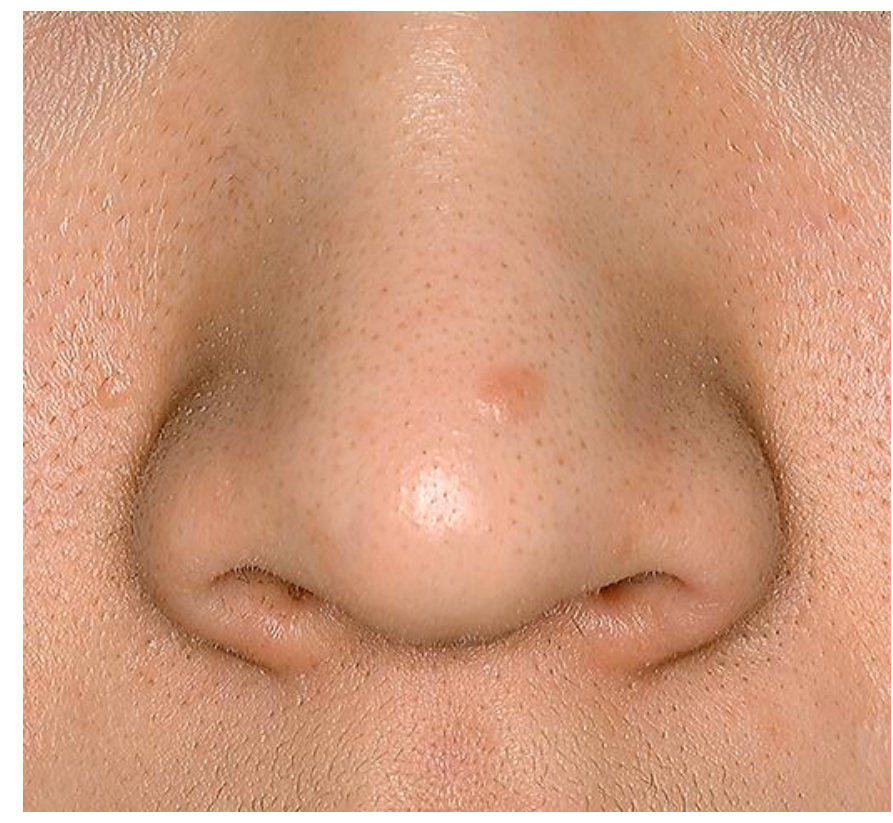

Fig. 1. A pinkish, dome-shaped papule (diameter $4 \mathrm{~mm}$ ) on the nose.

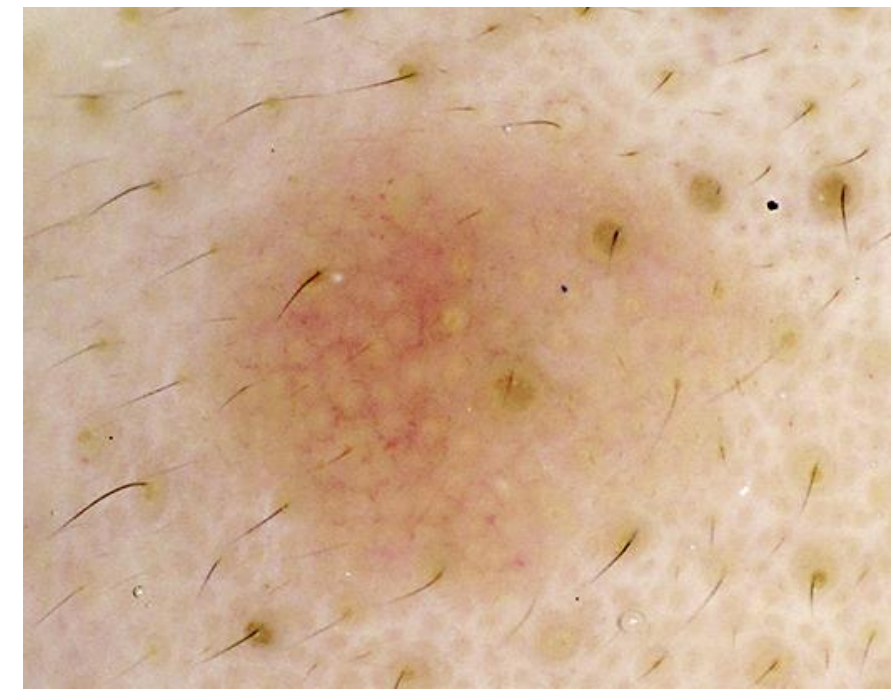

Fig. 2. Yellow globules surrounded by crown vessels/telangiectasias. No central follicular opening was present. Tiny scattered hairs were observed. 
Harada et al.: Hair Follicle Nevus with Sebaceous Hyperplasia: A Dermoscopic Observation
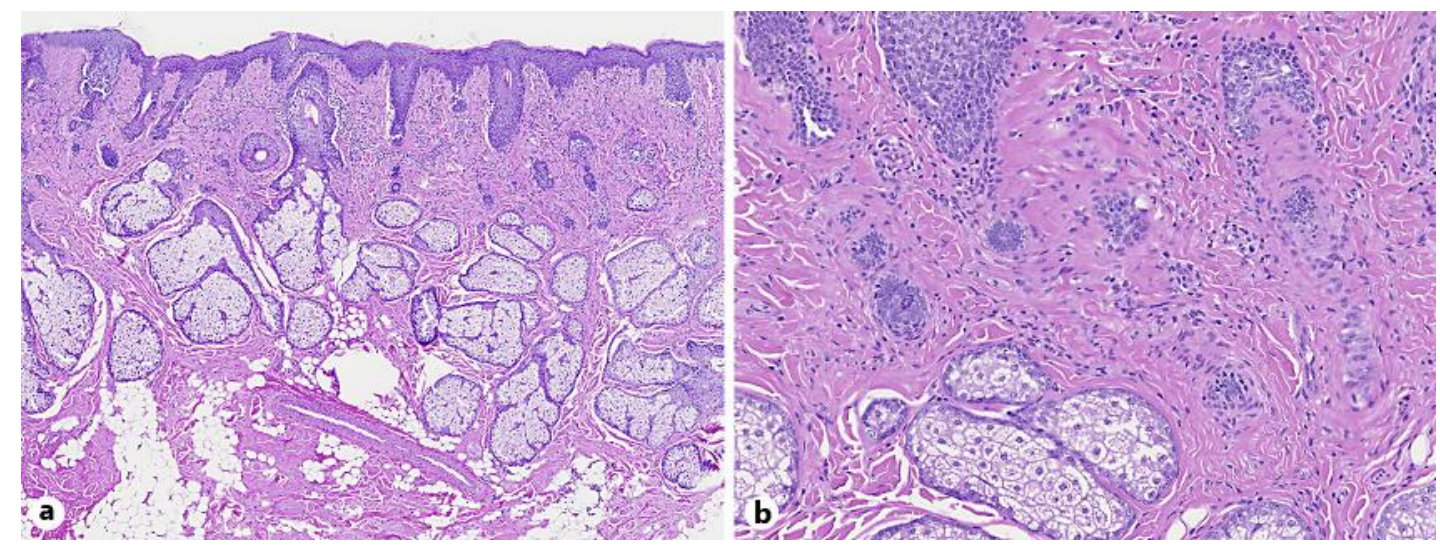

Fig. 3. a Well-differentiated vellus hair follicles in the upper dermis and hyperplasia of the sebaceous glands in the lower dermis (hematoxylin and eosin, $\times 100$ ). $\mathbf{b}$ Sebaceous glands were constituted of mature sebocytes (hematoxylin and eosin, $\times 200$ ). 